\title{
Comprehensive health workforce planning: re-consideration of the primary health care approach as a tool for addressing the human resource for health crisis in low and middle income countries
}

MICHAEL A. MUNGA ${ }^{1 *}$ and MUGHWIRA A. M WANGU²

${ }^{1}$ National Institute for Medical Research, P.O. Box 9653, Dar es Salaam, Tanzania

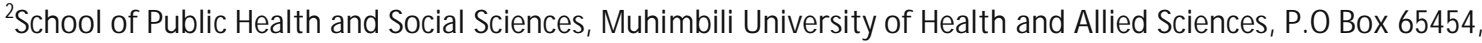
Dar es Salaam, Tanzania

\begin{abstract}
Although the Human Resources for Health (HRH) crisis is apparently not new in the public health agenda of many countries, not many low and middle income countries are using Primary Health Care (PHC) as a tool for planning and addressing the crisis in a comprehensive manner. The aim of this paper is to appraise the inadequacies of the existing planning approaches in addressing the growing HRH crisis in resource limited settings. A descriptive literature review of selected case studies in middle and low income countries reinforced with the evidence from Tanzania was used. Consultations with experts in the field were also made. In this review, we propose a conceptual framework that describes planning may only be effective if it is structured to embrace the fundamental principles of PHC. We place the core principles of PHC at the centre of HRH planning as we acknowledge its major perspective that the effectiveness of any public health policy depends on the degree to which it envisages to address public health problems multidimensionally and comprehensively. The proponents of PHC approach in planning have identified intersectoral action and collaboration and comprehensive approach as the two basic principles that policies and plans should accentuate in order to make them effective in realizing their pre-determined goals. Two conclusions are made: Firstly, comprehensive health workforce planning is not widely known and thus not frequently used in HRH planning or analysis of health workforce issues; Secondly, comprehensiveness in $\mathrm{HRH}$ planning is important but not sufficient in ensuring that all the ingredients of $\mathrm{HRH}$ crisis are eliminated. In order to be effective and sustainable, the approach need to evoke three basic values namely effectiveness, efficiency and equity.
\end{abstract}

Keywords: Primary health care approach, human resources, planning

\section{Introduction}

The crisis of human resource for health $(\mathrm{HRH})$ has different facets. It manifests itself in the form of acute deficits relative to needs, mal-distribution and inability to retain a motivated health workforce especially in underserved remote areas of many countries (Chomtiz et al., 1998; WHO 2000, 2004; Zurn et al., 2002; Dieleman et al., 2003; Dominic\& Kurowski, 2004; Dreesch et al., 2005; Dussault \& Franceschini, 2006; Glassman et al., 2008; Lehman et al., 2008).

The aim of this paper is to revitalise the discussion on the need for considering a comprehensive primary health care (PHC) approach in planning as a tool for addressing issues that compound the HRH crisis. It is argued that comprehensive and effective planning is at the core of all interventions designed to address the HRH crisis in many health systems around the world. Both the World Health Organization's framew ork on health systems strengthening and the 1978 Alma-Ata Declaration have considered the importance of comprehensive health service planning in the context of PHC, with a strong emphasis on the need to mobilise efforts and resources of other sectors for achieving better health outcomes in an equitable, efficient and effective way (WHO 2000, 2006).

Implementing comprehensive PHC planning is a complex endeavour as it requires extensive reforms in the health sector in order to create a 'responsive space' for other stakeholders to effectively participate in the planning process ( WHO, 2000, 2006). Since no country in the world has successfully managed to achieve such level of reforms in the health and other sectors, it is in our opinion that, PHC planning is fast becoming an 'all-encompassing term' as to be useless both from theoretical and policy point of views. For the purpose of this paper,

\footnotetext{
*Correspondence: M ichael A. Munga; E-mail: michaelmunga@yahoo.com
} 
however, the phrase is used to create an analytical context for the re-examination of its potential usefulness in planning and $\mathrm{HRH}$ policy development in order to address some of the aspects which substantially contribute to what is now known to be the HRH crisis. While the approach apparently looks rosy, scepticism has been prevalent among analysts on the feasibility of coordinating and putting together the efforts and resources of all important stakeholders in order to produce, attract and retain health workers in places where the needs are greatest and thus contribute to reversing the growing distributional imbalances. An attempt is made in the discussion section to level-off these potential worries from sceptics by emphasising that a stronger coordinating role of the ministries or departments of health is a prerequisite before embarking on implementing comprehensive HRH planning using the PHC approach.

For the purpose of this paper, HRH planning has been defined as a process of estimating the number of persons and kind of skills and attitudes they need to achieve predetermined health targets and ultimately health status objectives (Green, 1994). It involves the determination of who is going to do what, when, how and with what resources. Its main objective is to ensure that there is the right number of personnel, with the appropriate skills, at the right time with the minimum of waste. It is emphasized that such kind of planning must be continuous and not sporadic process and requires a continuous monitoring and evaluation (Green 1994).

Many reports in low and middle income countries have highlighted acute shortages of health workers who are outpaced by the actual needs for health care services (Anand \&Barnighaussen, 2004, 2007; WHO, 2006; Lehman et al., 2008; Madigan et al., 2008; Munga \& $M$ æstad 2009). Imbalanced distribution of the few er health workers available is also an important policy issue which has been attributed to failure by some employers to recruit the required health workers and retain them (Chomitz et al., 1998; WHO, 1998; Dambisya, 2002; Serneels et al., 2007; Munga et al., 2009). Related to this point a number of reports in Tanzania have indicated a skewed distribution of health workers whereby remote districts are disadvantaged by having a fewer number of health workers relative to their needs (Martineau, 2004; M uhondwa \& Fimbo 2006; Munga \& Mæstad, 2009). In addition to imbalanced distribution, declining morale and hence low productivity together with high attrition rates are few but consistently been cited as the factors compounding the health workforce crisis in Tanzania (Manongi et al., 2006; Meena 2009).

Over the past five decades or so, human resources planning in the health sector has generally been poor and thus resulted into a number of health personnel problems such as acute shortages, mal-distribution and poor performance (Green 1994; Nyoni et al., 2006). One of the chief causes of this situation is the fact that overall, planning for human resources for health in many countries had been developed in an ad hoc and fragmented manner (Dambisya et al., 2008). For example, the fact that health personnel training is largely confined to the education ministries/departments, it is unlikely that health planners will be able to influence medical training curricula, student intakes and outputs (Dambisya et al., 2008). In addition, in a situation where the ministries of finance of many developing countries are responsible for setting up public sector workers' salaries and controlling the wage bill, the ministries of health remain with virtually no or very limited influence on the number and type of health workers they need to employ, and how to design incentive packages (financial and non financial) to motivate employed health workers and ensure better performance (Dussault \& Dubois, 2003; Dussault \& Franceschini, 2006; Lehman et al., 2008; Dambisya et al., 2008). In the same token, many other central government organs equally exercise some amount of influence on HRH planning (Exhibit 1). In this case, Tanzania serves as a good example for extensive intervention of other central government bodies in matters (for example recruitment of health workers) which would hitherto be the responsibility of local governments especially in the current context of ongoing health sector reforms and decentralisation (Munga et al.,2009). While this paper does not wish to dishonour the importance of other sectors in health workforce planning, it emphasises the need for the ministries or departments of health in low and middle income countries to employ inter-sectoral collaboration for a comprehensive HRH planning which is result oriented. As discussed earlier on in this paper, the World Health Organization's two documents, namely Alma Ata Declaration and the Health 
Systems Strengthening Framework, explicitly uphold comprehensiveness and involvement of multi-sectoral stakeholders as important values in achieving health system's goals of achieving better health outcomes efficiently and equitably (WHO, 2000, 2006). It is in our opinion that, the use of comprehensive PHC approach in HRH planning (other than a selective, fragmented and health sector specific approach) is more likely to provide effective, long-term and sustainable solutions to many aspects of the HRH crisis in Tanzania and many low and middle income countries.

\section{Exhibit 1: Central government organs roles in HRH recruitment in Tanzania.}

While the law in Tanzania provides the mandate to districts to manage the recruitment of health workers, several central government departments continue to have key roles in the management of workers at the local government levels:

a) the Ministry of Finance approves districts' budgets and sets guidelines for the spending of locally mobilised financial resources as well as central government allocations,

b) the Public Service Department has a central role in approving employment permits and, in collaboration with the Public Service Commission, confirms health workers' employment and manages their promotions c) M inistry of Local Government administration approves transfers from one district to the other which has implications for their distribution between districts

d) Ministry of Health and Social Welfare directly administers pre-service training and staff recruitment for the regions and districts

\section{Materials and Methods}

Methodologically, this paper did not precisely follow the principles of a systematic literature review. It is rather a descriptive and narrative review of purposively selected literature and case studies from Tanzania and several other low and middle income countries. Relevant published literature on $\mathrm{HRH}$ and PHC including grey literature from the World Health Organization sources and other technical reports were reviewed. A wide range of literature on HRH and PHC were initially identified for a broad overview. Subsequently we focussed on those reports (published and grey literature) which were more relevant to our theme while excluding others. In terms of timeframe, all reports published between the 1990s and 2011 were considered relevant and included in the review. The time frame was deliberately chosen because most reforms and transformations in the health sector were occurring around this time, and a number of global milestones (Millennium Development Goals, Abuja Declaration, the Kampala Declaration, Ougadougou Declaration) in terms of achieving better health outcomes were set around this time. Similarly many of the milestones/goals dealing with improving HRH as espoused in different regional and global declarations, also featured prominently during this period. We considered a publication or report as relevant to our study only if: a) it discussed health personnel issues in middle or low income countries; b) it discussed the important HRH issues as raised in our conceptual framework (Figure 1); c) it discussed issues of planning in the health sector, particularly health personnel planning and finally; d) it discussed issues of PHC especially in relation to planning of health care services.

This descriptive and narrative review of literature was further nourished with a synthesis of expert opinions juxtaposed within the authors' extensive research experience in the area of HRH in Tanzania. We interviewed five experts in this field who were strategically consulted based on their proven record of evidence (their peer reviewed publications and technical/consultancy reports) that they are knowledgeable and are experienced analysts of issues surrounding human resource planning and primary health care; and the research projects they have conducted or participated in Tanzania and other Low Income Countries (LIC). The main focus of discussions with the experts was on their experiences regarding human resources planning and the overall resource allocation processes in the health sector. Of importance also, was to get their opinions on the challenges related to the involvement of many stakeholders in the HRH planning process. 
It is important to emphasise that the paper does not draw solely from primary data. That is the findings and arguments reported in this paper are largely from a descriptive review of literature with supplementary information from interviews with experts in the field. That is, it relies on available data in published scientific sources as well as grey literature and synthesis of expert opinions. In this and except for our experts whose consent was verbally sought, no ethical approval was required in the course of writing the paper. How ever the authors have ensured that views of other scholars and authors as used in this paper, have been correctly presented and acknowledged, and that the consulted experts were informed of the objectives of the consultations. The experts were also informed that their participation in the discussions was voluntary and they could withdraw from participating in the discussions at any time as they so wished. Finally the experts were assured that their information will be kept in strict confidence and no clues to their identities would be available to anybody or any institutions except the authors of this paper.

\section{Conceptual framework: Putting the Primary Health Care approach at the centre of HRH planning}

The central argument of our proposed conceptual framework is that planning, not just that pertaining to $\mathrm{HRH}$, may only be effective if it is structured to embrace the fundamental principles of PHC. We place the core principles of PHC at the centre of HRH planning as we acknowledge its major perspective that the effectiveness of any public health policy (and plans that ensue therefrom) depends on the degree to which it envisages to address public health problems multidimensionally and comprehensively. The proponents of PHC approach in planning have identified two basic principles that policies and plans should accentuate in order to make them effective in realizing their pre-determined goals. The principles are: (a) inter-sectoral action and collaboration; and (b) comprehensive approach in health policy development and planning (Biesma et al., 2009).

This paper focuses on these two principles. It is in our opinion that, failure to consider these elements when planning for production, recruitment/deployment, motivation and retention of health workers could result into unfavourable results such as ineffective interventions which, instead of addressing the root causes of the HRH crisis, they might help to preserve the 'status quo'. Thus, the main message of the proposed conceptual framework is that PHC approach in HRH planning may be more effective if it is implemented holistically whereby the key causes determining the $\mathrm{HRH}$ crisis are identified and analyzed in a systematic way by considering the complex inter-relationships between and among different actors and processes in the production of health workers, their recruitment and retention in places where they are mostly needed.

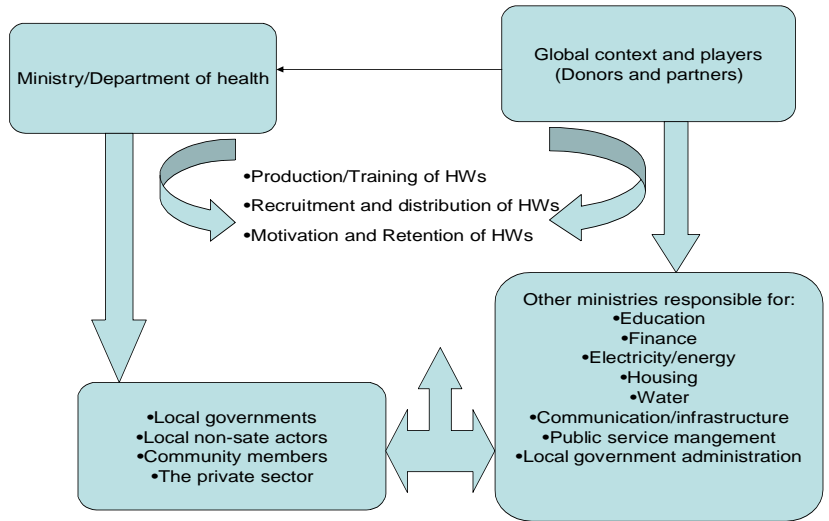

Figure 1: A simplified conceptual model of the interactions of stakeholders and important factors in comprehensive HRH planning using a PHC approach

We argue that the HRH management problems identified at the centre of our conceptual framew ork (Figure 1) cannot easily be solved by the policy actions of the health sector alone. That 
is, their solutions will require more fundamental interventions capturing the socioeconomic and political milieu (the context) of the health system in question in addition to the participation of other national and international players who have a stake in the health sector development (Biesma et al., 2009). For example the donors are important stakeholders if the goal of achieving comprehensive PHC health personnel planning is to be realistic (Figure 1) (Green 1994). We thus consider that their direct involvement (as it is for other sakeholders) in planning and implementation of the HRH management functions depicted, as innevitable. This consideration is in line with the Tanzanian M inistry of Health and Social Welfare's (M oHSW) series of annual joint health sector reviews. In all these reviews which are conducted anually, the issues of human respources crisis and particularly the need to tackle this by broadly involving all key sectors and actors, have had consistently been lifted up (MoHSW, 2010, 2011).

One of the most damaging effects of severely weakened and under-resourced health systems is the difficulty they face in producing, recruiting, and retaining health professionals, particularly in remote areas (Robinowitz et al., 2001; WHO 2006; Lehman et al., 2008; M unga et al., 2009; Wilson et al., 2009). Comprehensive planning to address the core aspects of HRH crisis (Figure 1) is in line with the call by the World Health Organization, which emphasizes the need to strengthen health care systems (WHO, 2000). One such effort in health systems strengthening is to ensure better strategies are in place in order to make certain that a motivated and skilled health workforce is recruited, distributed and retained in places where the health care needs are greatest. The subsequent sections describe the components of our proposed conceptual framework as they relate to production, recruitment, distribution, motivation and retention of health workers.

\section{The global context and its role and influences on human resource for health planning}

The conceptual Framework (Figure 1) highlights the importance of global contextual factors as crucial is shaping the HRH planning approaches and potentially dictating the nature of the interventions designed to address different aspects of the HRH crisis. The developed countries health care needs (and demand) and their competitive advantages in implementing active employment of additional health workers from low income countries is reported to have aggravated the problem of shortages in source countries, increased the costs of producing new (more) health workers to replace those who migrate to high income countries. In total, this has increased the loss due to limited returns from training and educating health workers (WHO, 2006; Bach, 2006; Kirigia et al., 2006).

The increased pace of globalization which goes hand in hand with a free flow of information about labour markets is believed to have added the momentum on the speed of health worker migration to developed countries (WHO, 2006; Bach, 2006; Kirigia et al., 2006). This tendency (migration) greatly weakens health systems in the countries where migrants originate (WHO, 2008). Migration of health workers from underdeveloped to developed health care labour markets has been (and still is) due to various pull and push factors (Nyoni et al., 2006; WHO, 2010b). The push factors are basically the unfavourable working environment existing in the majority of low income-cum-source countries. These include, but not limited to, low wages, poor housing conditions, poor working conditions and unattractive carrier paths. These conditions compel (push) trained health workers to move out of their home countries in search of greener pastures elsewhere, and thus leave their countries with an increased problem of shortage of health workers.

On the other hand the pull factors refer to the relatively attractive working conditions in the high income countries that tend to attract (pull) trained health workers from low income countries to high income countries (Nyoni et al., 2006; WHO, 2010; Taylor et al., 2011). These attractive environments are made even more effective by 'active' recruitment strategies of the recipient countries namely, high income countries (Nyoni et al., 2006; WHO 2010b; Taylor et al., 2011). It is thus logical to consider comprehensive PHC-HRH planning in the management of migration of health workers which substantially and negatively affect source countries. Even if 
migration of health workers to high income countries was not present, the low and middle income countries are already suffering from enormous shortages of health workers both in terms of numbers and skills. In many instances, these shortages are outpaced by expanding needs for health care services (Wyss, 2004).

We further argue that, if the efforts and resources of key actors (recipient and source countries) are comprehensively and proactively engaged, the thrust of negative effects (obviously, to source countries) of migration of health workers will somehow be mitigated and a win-win situation will most likely be created. Unfortunately and for many years, plans to fix many aspects of the health workforce crisis in low and middle income countries had either been inadequately or completely not considered the need to design compensation-based arrangements with the recipient countries to recover the losses incurred by the source countries. Since 2010, the World Health Organization has started discussions and consultations in order to put in place an international code of practice for the recruitment of health workers (WHO, 2010b). The code is expected to create a win-win situation between the source and the recipient countries. The essence of this code of practice is the acknowledgement by the international community that "highly trained and skilled" health personnel from the developing countries continue to immigrate at an increasing rate to more developed health care markets. It is in our opinion that, consideration of this code of practice in planning for $\mathrm{HRH}$ in low income countries is a step in the right direction towards incorporating the essential values of PHC in the HRH planning process. Whether or not this will eventually happen, is a matter of time for waiting because enforcement of this code does not legally bind any country. Rather, it depends on the voluntary decision of every country to participate or not.

\section{The role of donors}

With reference to our conceptual framework (Figure 1) donors or euphemistically called 'development partners', are equally important stakeholders if the goal of achieving comprehensive PHC health personnel planning is to be realistic (Green, 1994). We thus have an opinion that their direct involvement in planning and implementation of the HRH management functions, is innevitable. The proliferation of international health initiatives (such as Global Fund for HIV/AIDS, Tuberculosis and Malaria) and implementation of many donor-funded health programmes have created diverse concerns in many low and middle income countries regarding their impacts on the HRH production, recruitment, distribution and retention (World Bank, 1993,1995; Mtonya et al., 2005; Dambisya et al., 2008). It has been reported that the implementation of donor funded or internationally initiated and funded HIV/AIDS programmes have been recommended for contributing to the designing of innovative ways (e.g. Task shifting of clinical tasks from physician-clinicians to non-physician clinicians; and use of community health care workers) which are urgently needed to address the HRH shortages in the respective countries' health systems. In addition, the implementation of some Presidential Emergency Fund for Aids Relief (PEPFAR) activities in many low income countries has been going on hand in hand with designing interventions which focus on retaining health workers in underserved areas. For instance, physicians working in rural areas and remote areas have been provided with better working and living conditions such as housing, transportation, hardship allowances and educational stipends for their children (Dambisya, 2002; Dambisya et al., 2008; Sepúlveda et al., 2007). In Tanzania, the M kapa Foundation whose projects are implemented in the context of international donor initiatives, has introduced more or less similar innovations needed to attract and retain health workers in underserved remote areas which also have higher HIV/AIDS prevalence (Dambisya, 2002; Dambisya et al., 2008). Anecdotal evidence indicated that the M kapa Foundation's approach has been somewhat effective in addressing (albeit partially) the two aspects of the HRH crisis in Tanzania, namely recruitment and retention of health workers in underserved remote areas. However, sustainability of their approach is still questionable given that it is not institutionalised in the Tanzanian Ministry of Health and Social Welfare policy. In addition, there is no evidence indicating that the Ministry of Health and Social Welfare and other 
stakeholders have plans to scale up this intervention or contribute efforts and resources towards effective implementation of this initiative.

Implementation of the said international health initiatives and health related donor programmes and projects, are also known to have an enormous potential of negatively impacting on the health systems of poor countries, particularly their HRH base. In some of the Low and Middle Income Countries (LMICS) this tendency is reported to have implications for HRH availability, especially in the public sector whereby health workers 'follow money' by migrating to donor funded programmes/projects (Dambisya et al., 2008; Biesma et al., 2009). In some instances, the implementations of international health programmes have been blamed to have aggravated the shortage of health workers in many low income countries. It is reported in Malawi and Kenya that, selectively investing in global health initiatives or programmes for control of focal disease such as HIV/AIDS, Tuberculosis and M alaria is believed to have aggravated the shortage of qualified health workers as it increases the speed at which workers move from direct delivery of health services to go to work as programme managers' in Global Health Initiatives (GHI)-funded programmes (World Bank, 1995; M tonya et al., 2005).

In addition, the implementation of the World Bank and International Monetary Funds' (IMF) Structural Adjustment Programmes (SAPs) in the 1980s and 1990s has had serious impacts in the overall public sector human resources development. The policy prescription to cut government expenditure on social services (including health) has had negative repercussions on the production, recruitment, motivation and retention of health workers (World Bank, 1993, 1995; Dambisya, 2002; Bach, 2006). To date, these effects which started to ensue immediately after the implementation of the 1980-1990s IMF and the World Bank's conditionalities have not been adequately addressed by the existing interventions employed by health systems of many low and middle income countries.

Apparently, budget cuts in education and health sectors which has been one of the first World Banks' and IMF's conditionalities, entails that there would have been severe reduction of the capacity of training institutions to produce the required number of students who would qualify and probably be interested to take courses in the medical profession. This happened in Tanzania as it was in many aid-dependent low and middle income countries. In addition employment freeze and retrenchment of public servants (including health workers) which was another conditionality of the Breton's institutions (World Bank and IM F), was another blow to the Tanzanian health workforce base which was already poor in all known standards (Dominick \& Kurowski, 2004; Muhondwa \& Fimbo, 2006; Martineau, 2004 ). It meant that the fewer health workers who remained in health system started to experience increased workload, decreased morale and productivity and ultimately aggravated the thrust of shortages of health workers, particularly in underserved remote areas.

While the implementation of SAPs in Tanzania was holistically affecting the overall public service sector, the interventions set to address their ill-effects were poorly designed, were narrow and sector specific and above all, they are/were implemented piecemeal. It is in the same fashion that most of interventions to address the HRH crisis in the health sector were designed and implemented. Available evidence from several low and middle income countries indicate that the fewer health workers produced by the otherwise incapacitated training institutions are concentrated in urban areas (Chomitz et al., 1998; Dussaulut \& Franceschini, 2006; Lehman et al 2006; Serneels et al., 2007; Munga \& Mæstad, 2009). With this pattern, remote areas have consistently remained with fewer health workers than what is suggested by their share of diseases and public health burdens, probably due to poor deployment plans and ineffective retention strategies of those who agree to work in remote areas. We however do not have sufficient and strong evidence on whether medical training institutions' location (rural or urban) and their production capacity have any implications for the fairness in the distribution of health workers in low income countries such as Tanzania. 


\section{Ministry of Health coordination of other non-health sector actors}

It has been proposed in the preceding sections that, the ministry of health's role in the HRH planning is to centrally coordinate the efforts and resources of all actors in order to ensure that the key management functions (Figure 1) are effectively implemented. In Tanzania as it is for many countries, recruitment of health workers is practically not the domain of the health sector alone. One study has shown that the recruitment of health workers under the decentralised arrangement in Tanzania, involves many stakeholders from different sectors of the central government (M unga et al., 2009). The stakeholders have different roles in this process. The same study has shown that the decentralized recruitment systems creates an opportunity for health personnel planning at the district level to easily respond to needs of the communities residing in their areas of jurisdiction (M unga et al., 2009). In relation to PHC as a tool for planning, the same study has shown that integrating the strengths of central and decentralised government actors in the recruitment of health workers could create potentials for improving the recruitment and retention of health workers in underserved, remote areas. Perhaps what has not been emphasised before is the need for the ongoing health sector reforms to consider strengthening the coordinating role of the ministry of health in matters that aims to improve the systems' responsiveness to governance and $\mathrm{HRH}$ management functions which are portrayed in our conceptual framew ork in Figure 1

\section{Community members as important players in addressing some aspects of the HRH crisis}

Community participation as pointed out earlier is almost 'the head cornerstone' of the PHC approach in planning. For effectiveness and sustainability of interventions needed to address some or all aspects of the HRH crisis, it is imperative to involve (at some stages) community members in the planning and implementation of these interventions. The HRH literature has generally indicated that, health workers recognition by community members is one of the strong motivating factors for them to better perform and stay longer where they are deployed to offer the required services (Manongi et al., 2006; Lehman et al., 2008).

Community participation and decentralised planning processes are also recognised as key principles guiding all activities designed and implemented in the context of the PHC approach. However, existing modalities and practices of community involvement in planning and implementation of health related activities including planning for $\mathrm{HRH}$, had and still do not particularly show any major break-through. Under the existing decentralized system of governance, powers are still more concentrated at the district (council) levels and not so much on the communities. Attempts to empower communities to manage primary health care facilities particularly dispensaries and health centres, still have a long way to realize the desired results. It seems that, the professional gap between health workers and members of the community remains un-bridged and thus rendering participatory involvement of communities in the management of health facilities including addressing human resources, a distant reality in Tanzania and many other low income countries.

\section{Primary Health Care in relation to planning and ultimately alleviating the HRH crisis}

Having reviewed the literature on $\mathrm{PHC}$ and $\mathrm{HRH}$ planning, we can now create a link between comprehensive $\mathrm{PHC}$ planning in general and $\mathrm{HRH}$ planning in particular. Our conceptual framework (Figure 1) provides a descriptive overview of the interactions of important stakeholders in the HRH planning processes. The main link pin is borrowed from the World Health Organisations' health systems strengthening framew ork and the Alma-Ata declaration all of which emphasise that comprehensive and effective planning in the health service delivery and organisation depends largely on the involvement and participation of all important stakeholders within and outside the health sector (WHO, 1978, 2000). It is thus our opinion that PHC in HRH planning will create a platform whereby ministries/departments of health in the highly affected 
countries will engage in meaningful discussions and negotiations with stakeholders who control the production of health workers, those who control the financing, recruitment, distribution and retention of health workers.

Impliedly, the literature that has analysed the (potential) causes of the HRH crisis in many countries revealed that many of these causes are not within the domain of health sector and thus their alleviation may need comprehensive planning and multi-sectoral approach which are at the core of PHC as a tool for planning. The novel finding of this descriptive review is that $\mathrm{PHC}$ is neither a famous approach in HRH planning and policy development nor is it commonly used in the analysis of $\mathrm{HRH}$ issues. In addition, there seem some indications that countries such as Tanzania, have not been successfully in embracing the PHC approach in planning (not just that pertaining to $\mathrm{HRH}$ planning) for health services. Probably, one of the main reasons for this failure might be due to the tendencies by these countries to divergence form the Alma-Ata's emphasis in comprehensiveness and inter-sectoral collaboration; to selective, disjointed and sector based planning and implementation of health service programmes (WHO, 1978, 2008; Gillam, 2008). Note that we provide this conclusion of failed experience as we are not aw are of the existing best practices as far as intersectoral collaboration and comprehensiveness in planning are concerned. In the subsequent subsections, the paper briefly discusses the important aspects of the HRH crisis as earlier on shown in our proposed conceptual framew ork.

\section{The 'indifference' tow ards comprehensive PHC in HRH planning}

Except for the implementation of task-shifting to Community Health Workers (CHW) which has recently gained fame as one of the potential strategy to address the health workforce crisis affecting many Low and Middle Income countries, most of designed and implemented strategies to address the HRH crisis may literally be interpreted as stop-gap measures which cannot be sustained to offer long-term solutions (Munga et al., 2012). In addition, they have largely being 'health sector-specific' a phenomenon which is counterproductive to the vitals of PHC approach in planning. Schneider et al. (2007) have reported that scaled up CHW programmes are feasible, can lead to substantial health gains and they more often produce wider social benefits over a sustained period of time. In addition, reports indicate that health interventions delivered through or with the assistance of CHWs has the potential of not only addressing (albeit partially) the shortage of health workers but also institute a sense of community participation, partnership and ownership (WHO 2006, 2009; Ihuoma et al., 2009). These values are at the core of the expected outcomes of PHC approach in health policy and planning. However, in many low and middle income countries, these values seem to have, for many years, been neglected by policy makers in the field of HRH planning. We are not sure whether this negligence has been by default or by design.

\section{Strategies to address shortages of health workers}

Shortage of health workers has more often been linked to insufficient or inadequate production capacity of training institutions of many low and middle income countries. The World Health Report of the World Health Organization has argued that in order to maintain a reasonable balance of health workers in terms of numbers, diversity and competencies, there is a need for a thorough understanding of the driving forces and challenges that shape the education, the health systems and the labour markets (WHO, 2006). While this understanding may not be exhaustive or perfect, it emphasises two issues identified earlier. That is, comprehensiveness and multi-sectoral action. The experience in Tanzania indicates that health worker production planning is disjointed and is largely the domain of the following actors namely the Ministry of Education and Vocational Training (for Training of Medical Doctors and Specialists) and the Ministry of Health and Social Welfare for training of nurses, clinical officers and other midlevel health care professionals, famously known as allied professionals in the Tanzanian context. The Ministry of Finance is also critical in this partnership as all the sectoral budgets are essentially managed at this level. Of late, 
private training institutions have also emerged and are playing an important role in producing $\mathrm{HRH}$ at different levels. According to expert information, there is no one comprehensive plan that considers the priorities, efforts and resources of each important stakeholder and thus it is difficult to know how many health workers can be produced, how they can be produced and by what institutions, what skills are more important given the current situation, and so on. We thus argue that, although comprehensive PHC planning of HRH may sound unrealistic to many sceptics given the resource demands to coordinate all these efforts, a common understanding of how many and what types of health workers the country health system needs and how can they be produced is apparently crucial to all stakeholders and therefore their combined efforts are needed now than any time in the history of HRH planning.

\section{Recruitment and distribution of health workers}

To effectively recruit and distribute health professionals to where they are mostly needed, require cooperation of many players. An accurate interpretation of our conceptual model supports this argument. Poor working environment, inadequate and ineffective financial and non-financial incentives have made many low income countries fail to deploy health workers in places where the needs for health care are greatest (Robinson, 2007; VSO, 2009). How ever, many interventions designed or implemented to address these issues have largely been dominated by the ministries/departments of health. This contradicts with what is suggested by our hypothesis that $\mathrm{HRH}$ planning in the context of PHC offers the first right steps towards addressing the HRH crisis in a comprehensive manner.

As shown in exhibit 1, Tanzania for example, had tried different ways to increase the effectiveness of recruitment of health workers and thus increase their shares in remotely underserved areas. Centralised recruitment has almost dominated the recruitment and distribution processes for many years in Tanzania. In this context the main player for HRH planning has been the Ministry of Health and Social Welfare, in collaboration with other central bodies such as the Civil Service Department (CSD), the Public Service Commission, Ministry of Finance and the Ministry responsible for Local Governments and Regional Administration. With the introduction of health sector decentralisation reforms in the 1980s, local government authorities came up as an important player with a belief that HRH planning will be responsive to the actual needs of particular local government authorities as information about needs is more accessible at the local level than at the central level (Munga et al., 2009). The aim was also to institutionalise the concept of community participation which is also one of the central pillars of comprehensive PHC approach in HRH planning.

While recruitment was supposed to be implemented under the decentralised system, there has been a lot of uncoordinated stakeholder participation which created more confusion and delays in the recruitment process. What was essentially lacking in this multi-sectoral collaboration especially from central government bodies was stakeholder coordination, which is according to our conceptual model, is supposed to be the function of the M inistry of Health and Social Welfare. However, from 2006, Tanzania reintroduced partial centralisation of health worker recruitment in the context of the ongoing decentralisation reforms with the aim of speeding up the recruitment process especially that related to the recruitment of highly trained health workers. A study by Munga et al. (2009) has indicated that both strategies, decentralised and centralised recruitment system have strengths and weaknesses and thus, it is important to blend them by concentrating on their advantages while finding ways to alleviate their weaknesses.

One 'perceived' advantage of centralised recruitment system is that, it is capable of recruiting and distributing highly qualified health personnel and deploy them in even those places 'stigmatised' as unattractive, although underserved. Centralised recruitment on the other hand, was perceived to not be effective in retaining health workers where they are deployed. Decentralised recruitment on the other hand, was perceived to be effective in attracting lower cadre health personnel from within a particular district or nearby districts and was also perceived to be effective in retaining them Munga et al. (2009). This could apparently be due to the fact 
that, the employed health workers are either aware or used to the working and living environment of the places where they are supposed to be working, which is a crucial piece of information before they make their decisions to accept or reject an employment offer (M unga et al., 2009).

\section{Retention of health workers}

Several interventions have been applied, in different settings, to attract, recruit and retain and ultimately increase the shares of health workers in areas described as remote and underserved. The literature has frequently labelled these interventions as selection, education, coercion, and incentives. Selection interventions focus on recruiting students with a high probability of choosing a remote working place into medical training programmes. Educational interventions concentrate on strategies that optimise medical training programmes in order to stimulate prospective health workers to locate their practice in remote areas. Coercive interventions focus on the use of authoritarian methods by institutions such as medical councils, professional bodies and employers to force health workers into compulsory rural practice for a specified period of time after their graduation. Incentives are always implemented as financial or non-financial interventions applied to induce health workers to accept working in remote underserved areas, improve productivity or to reduce the rate at which health workers will migrate to other labour markets within or between countries (Dussault \& Franceschini, 2006; Wilson et al., 2009).

Retention of health workers in their work positions is known to be a function of many factors. Existing literature suggests that among the factors that influence health workers decisions to remain in their jobs or to migrate, are their working and living conditions or environment, financial incentives, carrier development, their recognition and several others (Robinson, 2007; Willis-Shattuck et al., 2008; WHO, 2009). At the global level the World Health Organization has come up with a comprehensive package of recommendations on how to improve retention of health workers especially in rural poor settings (WHO, 2010). Tanzania like other countries is also faced with this challenge of retaining health staff and several isolated measures have been taken to deal with it. Related to this, there is no sufficient and contextspecific evidence on the costs and effectiveness of these interventions, especially in poorly resourced settings like Tanzania. It is notew orthy that, even with such evidence, this problem should not be seen to solely concern the Ministry of Health and hence, it has to be addressed in a comprehensive manner by involving other key stakeholders is as much as it is required and as prescribed in the major principles guiding PHC approach in HRH planning (Figure 1).

Given the complex nature of the HRH retention, it is suggested that a country may select one or bundles of these interventions (given availability of context-specific evidence of feasibility and cost effectiveness). However, a key factor to implementation success of these interventions is related to the extent to which important stakeholders can be engaged; and how these multiple actors who have a stake in HRH planning can be engaged. Examples of these stakeholders are such as training institutions and universities, health and educational ministries, civil service departments, professional associations, non-governmental organizations and others. In relation to this point, another equally important factor is how to harmonise the stakeholders' interests and resources that each stakeholder bring to bear in designing and implementing these interventions (WHO, 2006, 2010; Chen, 2010).

Although decentralised recruitment system does not feature in our conceptual framework, it is a key component in PHC-HRH planning. One study in Tanzania has compared centralised and decentralised recruitment of health workers (Munga et al., 2009). Although this has not been made so explicit and direct in the reffered study above, it is in our opinion that the working together of local and central government stakeholders in the HRH recruitment processes is at the core of PHC in HRH planning. The study by Munga et al. (2009) has shown that recruitment of health workers under a decentralised arrangement has not only been characterised by complex bureaucratic procedures, but by severe delays and sometimes failure to get the required health workers. Further, the study has revealed that recruitment of highly skilled 
health workers under decentralised arrangements may be both very difficult and expensive. In the same study, decentralised recruitment was perceived to be more effective in improving retention of the lower cadre health workers within the districts.

In contrast, the centralised arrangement was perceived to be more effective both in recruiting qualified staff and balancing their distribution across districts, but poor in ensuring the retention of employees (Munga et al., 2009). The study has concluded that, a combination of centralised and decentralised recruitment represents a promising hybrid form of health sector organisation in managing human resources by bringing the benefits of two worlds together. The study has concluded further that, in order to ensure that the potential benefits of the two approaches are effectively integrated, careful balancing defining the local-central relationships in the management of human resources needs to be worked out (M unga et al., 2009). Synergising these benefits and working to do away with the deficiencies of each recruitment system is actually what is advocated by the main principles of PHC as a tool for health services planning.

\section{Conclusion}

The analysis presented in this review intended to inform policy stakeholders and particularly governments, to think more creatively about the effective approaches in HRH planning and policy development strategies which are capable of addressing different aspects of health worker crisis facing many countries in the world. While the present analysis might have added some knowledge needed to inculcate the stakeholders' enthusiasm to take proactive action, the generated information may not be sufficient enough to help policy makers focus their attention on comprehensive approaches for alleviating the HRH crisis. This is because the conceptual and analytical foundations of many analyses found in the literature have had an implicit assumption that HRH problems have originated largely from the 'malfunctioning' of the health sector and thus most of the proposed interventions responding to these problems were by default or design health-sector-focused. This is untenable both for policy development and planning for HRH. Related to this aspect, this descriptive review provides an indication that the PHC is neither a wellknown approach in HRH planning and policy development nor is it frequently used in the analysis of $\mathrm{HRH}$ issues. This is true both in the theoretical and policy literature

PHC in HRH planning is neither popular in the reviewed literature nor given its due attention in the day to day practice of HRH planning, notwithstanding the fact that many policy and programme documents of the ministries of health (for example in Tanzania) claim to uphold their noble values namely, comprehensiveness and multi-sectoral cooperation in planning and programme implementation. How ever, the analysis presented in this paper has so far emphasised the importance of $\mathrm{PHC}$ as a tool for policy development and planning for $\mathrm{HRH}$. It is thus proposed that the ministries of health in low and middle income countries facing the HRH crisis of one form or the other need to enter, with enthusiasm, into partnerships with other sectors and agencies (nationally and internationally) and communities to develop inter-sectoral policies which address the key determinants of the crisis in all its different manifestations. Selective approaches (as opposed to comprehensive ones) which are largely health sector specific may not provide longer term and sustainable solutions to the HRH crisis which apparently, seems to grow and outpaces the efforts to deal with it.

Having been designed following the important principles of PHC, interventions responding to the $\mathrm{HRH}$ crisis must invoke three basic criteria, namely effectiveness, efficiency and equity. As regards to effectiveness, the scale of the response to any aspect of the HRH crisis must be commensurate with the challenge posed. That is, if for example the objective is to increase the share of health workers to work in underserved and 'stigmatised' remote areas and retain them there, then the interventions aimed to achieve this objective should not only be implemented properly and in time but also they should be seen that they produce the intended results in comparison to the existing interventions or in comparison to doing nothing. In terms of efficiency, researchers and policy makers must advocate and implement interventions that are evidently known to be capable of keeping down the costs of implementation. Equity is the concern that 
policy makers and implementers of interventions need to take to heart because in many cases, the starting point for taking action may unintentionally be deeply inequitable. For example, implementing some re-distributive interventions designed to increase the share of health workers in remote undeserved areas (if distributional inequalities of the $\mathrm{HRH}$ is the key issue to be addressed) may end up punishing some communities that from a face value, seem to have more health workers than they need. In this case a careful and comprehensive health care needs analysis must inform the design and implementation of these interventions. This again needs to be done by respecting the principles of comprehensiveness and inter-sectoral collaboration

Obviously, implementation of PHC-based health workforce planning and its sustenance is challenging and critically require resources such as finances, expertise and skills which are not sufficiently present in many low and middle income countries. In addition, its implementation may be challenged by enormous needs for coordination of diverse stakeholders' interests, and priorities in the context of constrained resource base. Thus, coherent and integrated partnership of the ministries/departments of health with other stakeholders is needed in order to pull all the available resources from all stakeholders in order to achieve a common goal. In this collaboration, the ministries of health can take the facilitator role and a 'central' coordinating role. In addition, the involvement of communities in addressing various aspects of health sector development (such as health workforce crisis) needs to be practised with much more devotion and focus. Community involvement in HRH planning can be done in many ways but in this paper, we propose two: (i) in the context of difficulties of retaining health workers in remote areas, community members residing in these areas can directly be involved to design context specific interventions of retaining health workers. Given the fact that they are used to the lives of these areas, their knowledge on 'retention support factors' can be taped through consulting them and involving them in the initial processes of developing health worker retention strategies; (ii) Community members can in a better way, contribute in the planning of how they can be involved as direct health providers and thus contributing to addressing the shortage of health workers in rural and remote areas.

\section{Acknowledgements}

The authors would like to thank their respective institutions for providing conducive environment which helped the authors to conceptualise and eventually write this paper. We are also grateful to our experts in the field whose educated opinions during our discussions have substantially contributed to strengthening our arguments. However, the views expressed in the paper are solely those of the authors and they do not represent in any way the views or positions of the institutions for which authors are affiliated. Any use of trade or organisation names is for identification purposes only and does not imply endorsement by the authors.

\section{References}

Anand, S. \& Barnighausen, T. (2004) Human resources and health outcomes: cross-country econometric study. Lancet 364, 1603-1609.

Anand, S. \& Barnighausen, T (2007) Health workers and vaccination coverage in developing countries: an econometric analysis. Lancet 369, 1277-1285.

Bach, S. (2006) International mobility of health professionals: Brain drain or brain exchange? Discussion Paper No. 2006/82. United Nations University. The World Institute for Development Economics Research, Helsinki.

Biesma, R.G., Brugha, R., Harmer, A., Walsh, A., Spicer, N. \& Walt, G. (2009) The effects of global health initiatives on country health systems: a review of the evidence from HIV/AIDS control. Health Policy and Planning 24, 239-252.

Chen, L.C. (2010) Striking the right balance: health workforce retention in remote and rural areas. Bulletin of the World Health Organisation 88, 1 
Chomitz, K.M., Setiadi, G., Azwar, A., Ismail, N. \& Widiyarti, (1998) What doctors want?: developing incentives for doctors to serve in Indonesia's rural and remote areas. The World Bank, Washington DC, USA.

Dambisya, Y.M., Modipa, S., Nyanzema, N. et al (2008) A review on the impact of HIV and AIDS programmes on health worker retention: EQUINET Discussion Number 71

Dambisya, Y.M. (2007) A review of no-financial incentives for health worker retention in east and southern Africa. EQUINET Discussion Paper Number 44.

Dieleman, M., Cuong, P., Anh, L. \& Martineau, T. (2003) Identifying factors for job motivation of rural health workers in North Viet Nam. Human Resources for Health 1, 10.

Dominic, A. \& Kurowski, C. (2004) Human resources for health: An appraisal of the status quo in Tanzania Mainland. M inistry of Health, Tanzania \& World Bank.

Dreesch, N., Dolea, C., Dal Poz, M.R., Goubarev, A., Adams, O., Aregawi, M., Bergstrom, K., Fogstad, H., Sheratt, D., Linkins, J., Scherpbier, R. \& Youssef-Fox, M. (2005) An approach to estimating human resources requirements to achieve Millenium Development Goals. Health Policy and Planning 20, 267-276.

Dussault, G. \& Dubois, C.A. (2003) Human resources for health policies: a critical component in health policies. Human Resources for Health $1: 1$

Dussault, G. \& Franceschini, M. (2006) Not enough there, too many here: understanding geographical imbalances in the distribution of the health workforce. Human Resources for Health 4:12.

Glassman, A., Becker, L., Makinen, M. \& De Ferant. D. (2008) Planning and costing human resources for health. Lancet 371, 693-695.

Green, A. (1994) An Introduction to Health Planning in Developing Countries. Oxford University Press, New York, USA.

Ihuoma, E. (2009) Task Shifting: A strategic response to the health care human resources crisis. A qualitative study of hospital based HIV clinics in northern-central Nigeria. http//www.stti.iupui.edu/pp07/congress/ihuoma_e.pd. 2009.

Kirigia, J., Gbary, A., Muthuri, L., Nyonim J . \& Seddoh, A. (2006) The cost of health professionals' brain drain in Kenya. BMCHealth Services Research 6:89.

Lehmann, U., Dieleman, M., Martineau, T. (2008) Staffing remote rural areas in middle- and lowincome countries: A literature review of attraction and retention. BMC Health Services Research 8:19.

Madigan, E., Curet, O. \& Zrinyi, M. (2008) Workforce analysis using data mining and linear regression to understand HIV/AIDS prevalence patterns. Human Resources for Health 6:2.

M anongi, R.N., Marchant, T.C. \& Bygbjerg, I.C. (2006) Improving motivation among primary health care w orkers in Tanzania: a health worker perspective. Human Resources for Health 4:6.

Martineau, T. (2004) Report of the study on human resources for health governance in Tanzania for the Ministry of Health. Ministry of Health, Dar es Salaam, Tanzania/World Bank.

Meena, R. (2009) Paid care workers inTanzania: a general description of nurses and home base care givers. United Nations Research Institute for Social Development (UNRISD), Research Report 4, Geneva, Switzerland.

MoHSW (2010) Annual J oint Health Sector Review. Ministry of Health and Social Welfare, United Republic of Tanzania.

MoHSW (2011) Annual J oint Health Sector Review. Ministry of Health and Social Welfare, United Republic of Tanzania.

M tonya, B., M wapasa, V. \& Kadzandira, J . (2005) System-wide effects of the Global Fund in Malawi: Baseline Study Report. The Partners for Health Reformplus, Abt Associates Inc., Bethesda, MD, USA.

Muhondwa, E. \& Fimbo, B. (2006) Impact of HIV/AIDS on Human Resources for Health in Tanzania. Assessment report submitted to the Ministry of Health and Social Welfare and ECSA Secretariat, Arusha, Tanzania.

Munga, M.A. \& Mæstad, O. (2009) Measuring inequalities in the distribution of health workers: the case of Tanzania. BMCHuman Resources for Health 7:4. 
Munga, M.A., Songstad, N.G., Blystad, A. \& Mæstad, O. (2009) The decentralisation-centralisation dilemma: recruitment and distribution of health workers in remote districts of Tanzania. BMC International Health and Human Rights 9 (9).

Munga, M.A., Kilima, S.P., Mutalemwa, P.P., Kisoka, W.J . \& Malecela, M.N. (2012) Experincences, opportunities and challenges of implementing task shifting in underserved remote settings: the case of Kongwa district, central Tanzania. BMC International Health and Human Rights 12 (27).

Nyoni, J., Gbary, A., Awases, M., Ndecki, P. \& Chatora, R. (2006) Policies and plans for HRH: Guidelines for countries in WHO Africa Region. World Health Organization, Brazzaville, Republic of Congo.

Rabinowitz, H.K., Diamond, J.J., Markham, F.W. \& Paynter, N.P. (2001) Critical factors for designing programs to increase the supply and retention of rural primary care physicians. JAMA 286, 1041-1048.

Robinson, R. (2007) The costs and benefits of health workers migrationfrom east and southern Africa (ESA). A Literature Review. Regional Network for Equity in Health in East and Southern Africa (EQUINET). Discussion Paper No. 49. http//www .equinetafrica.org/bib/docs/DIS49HRrobinson.pdf.

Schneider, H., Hlophe, H. \& van Rensburg, D. (2008) The effects of global health initiatives on country health systems: a review of the evidence from HIV/AIDS control. Health Policy and Planning 23, 179-187.

Sepúlveda, J., Carpenter, C., Curran. J., Holzemer, W., Smits, H., Scott, K. \& Orza, M. (2007): PEPFAR Implementation: Progress and Promise. Institute of Medicine of the National Academies. The National Academies Press, Washington, DC-USA

Serneels, P., Lindelow, M., Garcia-Montalvo, J. \& Barr, A. (2007) For public service or money: understanding geographical imbalances in the health workforce. Health Policy and Planning 22, 128-138.

Taylor, A.L., Hwenda, L., Larsen, B. \& Daulaire, N. (2011) Stemming the Brain Drain - A WHO Global Code of Practice on International Recruitment of Health Personnel. New England J ournal of Medicine 365, 2348-2351

VSO (2009) Brain Gain Making Health Worker Migration Work for Rich and Poor Countries: The Perspective from Africa. VSO Briefing. http//www.vso.org.uk/lmages/Brain_gain_tcm7928869.pdf. 2009.

WHO (1978) The Alma Ata Declaration. The World Health Organization, Geneva, Switzerland

WHO (1998) The Challenges of implementation- District health systems for Primary Health Care. World Health Organization, Geneva, Switzerland.

WHO (2006) The World Health Report-"Working Together for Health". The World Health Organization, Geneva, Switzerland

WHO (2008) International recruitment of health personnel: draft global code of practice. Report of the Secretariat of the World Health Organization, Geneva, Switzerland.

WHO (2010) Increasing access to health workers in remote and rural areas through improved retention. The World Health Organization's Background paper. Geneva, Switzerland.

WHO (2010) The Global Code of Practice on International Recruitment of Health Personnel. $63^{\text {rd }}$ World Health Assembly Agenda 63.16.

WHO (2008) The World Health Report: Primary Health Care-now more than ever. The World Health Organization, Geneva-Switzerland.

WHO(2000) The World Health Report: Health Systems: Improving performance. World Health Organization, Geneva, Switzerland.

Willis-Shattuck, M., Bidwell, P., Thomas, S., Wyness, L., Blaauw, D. \& Ditlopo, P. (2008) Improving motivation and retention of health professionals in developing countries: a systematic review. BMC Health Services Research 8: 247.

Wilson, N.W., Couper, I.D., De Vries, E., Reid, S. \& Fish, T. (2009) A critical review of interventions to redress the inequitable distribution of health care professionals to rural and remote areas. Rural and Remote Health 9. 
World Bank (1993) Investing in health. In: World Development Report. Oxford: Oxford University Press.

World Bank (1995) Better Health in Africa: experiences and Lessons Learned. The World Bank, Washington DC, USA.

Wyss, K. (2004) An approach to classifying human resources constraints to attaining healthrelated Millennium Development Goals. Human Resources for Health 2:11

Zurn, P., Dal Poz, M , Stillwell, B. \& Adams, O. (2002) Imbalances in the Health Workforce: Briefing Paper. The World Health Organization, Geneva, Switzerland. 